\title{
Evaluation of the antimicrobial susceptibility testing process in clinical microbiology laboratories at Niamey, Niger
}

\author{
${ }^{1}$ Idrissa, H., *2Abdoulaye, O., ${ }^{3}$ Yacouba, A., ${ }^{3}$ Alhousseini Maiga, D., ${ }^{1}$ Moumouni Sambo, H., \\ ${ }^{4}$ Doutchi, M., ${ }^{5}$ Chaibou, S., ${ }^{6}$ Sani, O., ${ }^{3}$ Mamadou, S., and 7 Sangare, L. \\ ${ }^{1}$ Direction des Laboratoires de santé (DLS), Ministère de la santé publique, Niger \\ ${ }^{2}$ Faculté des Sciences de la Santé, Université Dan Dicko Dankoulodo, Maradi, BP 465, Niger \\ ${ }^{3}$ Faculté des Sciences de la Santé, Université Abdou Moumouni, Niamey, Niger \\ ${ }^{4}$ Faculté des Sciences de la Santé, Université de Zinder, Niger \\ ${ }^{5}$ Hôpital Général de Référence de Niamey, Niger \\ ${ }^{6}$ Centre de Recherche Médicale et Sanitaire, Niger \\ 7Unité de Formation et de Recherhe en Sciences de la Santé (UFR/SDS), Université Joseph Ki-Zerbo, \\ Ouagadougou, Burkina Faso \\ *Correspondence to: ousmaneabdoulaye2010@yahoo.com; +227 96354580
}

\begin{abstract}
:
Background: Risk assessment is the means of identifying and evaluating potential errors or problems that may occur in testing process. The aim of this study was to perform risk assessment of antimicrobial susceptibility testing (AST) process in clinical microbiology laboratories of Niamey, Niger Republic.

Methodology: We conducted a descriptive cross-sectional study from October 1 to December 31, 2019, to evaluate AST performance in seven clinical microbiology laboratories at Niamey, the capital city of Niger republic. The evaluation focused on the determination of the criticality index (CI) of each critical point (frequency of occurrence of anomalies, severity of the process anomaly, and detectability of the anomaly during the process) in the AST process and the performance of the AST through an observation sheet using two reference strains; Escherichia coli ATCC 25922 and Staphylococcus aureus ATCC 29213.

Results: The criticality index (CI) was greater than 6 for most of the critical points related to material, medium, equipment, method and labour for the AST process in all the laboratories. A range of $18-100 \%$ errors on the inhibition zone diameters of the reference strains were observed. Major and/or minor categorization (Sensitive S, Intermediate I and Resistance R) discrepancies were found at all the laboratories for either one or both reference strains. The antibiotics most affected by the S/I/R discrepancies were trimethoprim (100\%), vancomycin (100\%), amoxicillin (80\%) and amoxicillin + clavulanic acid (70\%).

Conclusion: This study showed a deficiency in the control of critical control points that impacts the performance of the AST reported by the laboratories in Niger. Corrective actions are needed to improve the performance of AST in clinical microbiology laboratories in Niger.
\end{abstract}

Keywords: Quality control; antibiogram; criticality index; clinical microbiology laboratories; Niger

Received Jun 28, 2021; Revised Aug 13, 2021; Accepted Aug 14, 2021

Copyright 2021 AJCEM Open Access. This article is licensed and distributed under the terms of the Creative Commons Attrition 4.0 International License $<$ a rel="license" href="http://creativecommons.org/licenses/by/4.0/", which permits unrestricted use, distribution and reproduction in any medium, provided credit is given to the original author(s) and the source. Editor-in-Chief: Prof. S. S. Taiwo

\section{Evaluation du processus de réalisation de l'antibiogramme dans les laboratoires d'analyses de biologie médicale de la ville de Niamey, Niger}

\footnotetext{
${ }^{1}$ Idrissa, H., ${ }^{* 2}$ Abdoulaye, O., ${ }^{3}$ Yacouba A., ${ }^{3}$ Alhousseini Maiga, D., ${ }^{1}$ Moumouni Sambo, H., ${ }^{4}$ Doutchi, M., ${ }^{5}$ Chaibou S., ${ }^{6}$ Sani, O., ${ }^{3}$ Mamadou, S., et ${ }^{7}$ Sangare, L.
}

${ }^{1}$ Direction des Laboratoires de santé (DLS), Ministère de la santé publique, Niger

${ }^{2}$ Faculté des Sciences de la Santé, Université Dan Dicko Dankoulodo, Maradi, BP 465, Niger

${ }^{3}$ Faculté des Sciences de la Santé, Université Abdou Moumouni, Niamey, Niger

${ }^{4}$ Faculté des Sciences de la Santé, Université de Zinder, Niger

${ }^{5}$ Hôpital Général de Référence de Niamey, Niger

${ }^{6}$ Centre de Recherche Médicale et Sanitaire, Niger 
${ }^{7}$ Unité de Formation et de Recherhe en Sciences de la Santé (UFR/SDS), Université Joseph Ki-Zerbo, Ouagadougou, Burkina Faso

*Correspondence à: ousmaneabdoulaye2010@yahoo.com; +22796354580

\section{Résumé:}

Contexte: L'évaluation des risques est le moyen d'identifier et d'évaluer les erreurs ou les problèmes potentiels qui peuvent survenir dans le processus de test. L'objectif de cette étude était de réaliser une évaluation des risques du processus d'antibiogramme $(A B G)$ dans les laboratoires de microbiologie clinique de Niamey, en République du Niger.

Méthodologie: Nous avons mené une étude transversale descriptive du 1er octobre au 31 décembre 2019 pour évaluer la performance des ABG dans sept laboratoires de microbiologie clinique à Niamey, capitale de la république du Niger. L'évaluation a porté sur la détermination de l'indice de criticité (IC) de chaque point critique (fréquence d'apparition des anomalies, gravité de l'anomalie du processus et détectabilité de l'anomalie au cours du processus) dans le processus et la performance des AGB à travers une fiche d'observation en utilisant deux souches de référence; Escherichia coli ATCC 25922 et Staphylococcus aureus ATCC 29213.

Résultats: L'indice de criticité était supérieur à 6 pour la plupart des points critiques liés au matériel, au milieu, à l'équipement, à la méthode et à la main-d'œuvre pour le processus AST dans tous les laboratoires. Une fourchette d'erreurs de 18 à $100 \%$ sur les diamètres des zones d'inhibition des souches de référence a été observée. Des écarts de catégorisation majeurs et/ou mineurs (Sensible: S, Intermédiaire: I et Résistance: R) ont été constatés dans tous les laboratoires pour l'une ou les deux souches de référence. Les antibiotiques les plus touchés par les écarts S/I/R étaient la triméthoprime $(100 \%)$, la vancomycine $(100 \%)$, I'amoxicilline $(80 \%)$ et l'amoxicilline + acide clavulanique (70\%).

Conclusion: Cette étude a montré une déficience dans le contrôle des points de contrôle critiques qui a un impact sur la performance de l'antibiogramme rapportée par les laboratoires au Niger. Des actions correctives sont nécessaires pour améliorer la performance des ABG dans les laboratoires de microbiologie clinique au Niger.

Mots clés: Contrôle de qualité, antibiogramme; indice de criticité; laboratoires de microbiologie clinique; Niger

\section{Introduction:}

Antibiotic susceptibility test (AST) is a common test in clinical microbiology laboratory. AST is a critical component of a quality control plan to ensure reliable patient results and management of bacterial infections. AST results guide clinicians to prescribe appropriate antibiotics for treatment by testing the effectiveness of antimicrobial drugs against specific organisms. Numerous methods are used to perform AST in clinical laboratory, but the Kirby-Bauer disk diffusion test has long been widely used for evaluating the susceptibility of specific organisms to antimicrobial drugs (1). Different actors are involved in the process of performing an AST using the Kirby Bauer method and each of them carries error risk that can directly affect the results. Risk assessment is the means of identifying and evaluating potential errors or problems that may occur in testing process. The fishbone diagram, also known as the Ishikawa diagram, identifies five components that must be evaluated in risk assessment that includes the specimen, the test system, the reagents, environment, and testing personnel (2).

Risk assessment can be used in AST as a brainstorming session that immediately sorts ideas into the potential source of error and risk factor. It can provide to clinical laboratory the basis for taking action to manage the negative consequences of unreliable AST. It is for this reason, that internal quality control (IQC) should be performed regularly with reference strains (3). In Niger, few clini- cal bacteriology laboratories control the quality of their AST process. The objective of this study was therefore to perform risk assessment of the AST process in clinical microbiology laboratories of Niamey, Niger.

\section{Materials and method:}

\section{Study design}

We conducted a descriptive crosssectional study from October 1 to December 31,2019 to evaluate the quality of the AST process in public and private clinical microbiology laboratories where bacteriological analysis and AST were performed at Niamey, the capital city of Niger republic. This design allowed the evaluation of the critical points and the process of carrying out AST in each selected laboratory.

\section{Selection of laboratories}

In this study, all laboratories in the city of Niamey that perform bacteriology analyses were eligible but the inclusion criterion was availability of bacteriology examinations particularly AST. A total of 7 eligible laboratories were selected; 3 from the public and 4 from the private sector.

Evaluation of the process and procedure of AST and determination of criticality index

The critical points and the measures taken to control the risks were analyzed according to the 5-M Model (Man, Medium, Machine, Management and Mission) method (4), and then checked onsite using a FMECA (Failure Mode, Effects and Criticality Analysis) 
type observation sheet to determine the sources of error $(3,5)$. The criticality index (CI) of each point was evaluated on a scale of three items; Frequency ( $F$ ) of occurrence of anomalies, Severity (G) of the process anomaly, and Detectability (D) of the anomaly during the process. Each item was rated from 1 to 3 according to the 2017 adapted first edition of QUAMIC (2), which allowed the establishment of a criticality index (CI) from multiplication of the 3 items i. e. criticality index $(\mathrm{CI})=\mathrm{F} \times \mathrm{G} \times \mathrm{D}$, and the threshold cut off was $\mathrm{CI} \geq 6$.

The evaluation of the performance of the AST process was an experimental verification onsite, according to the procedure for carrying out AST established by each laboratory using supplied reference strains. These AST processes were, among others, samples and reagents, temperature of conservation and that of the laboratory rooms, working materials, methods used, technical integrity of reagents and competence of the staff. The relevant parameter verified was the accuracy of the results. For this purpose, two reference strains (S1: Escherichia coli ATCC 25922 and S2: Staphylococcus aureus ATCC 29213) were made available to the laboratories on a blinded basis. Each laboratory (LBM1 - LBM7) performed AST on each of the reference strains after identification according to the procedure in use in the laboratory and under the same technical conditions as the clinical samples, but no clinical strains were involved in the study.

\section{Results:}

A total of 8 laboratories in the city of Niamey were selected but only 7 (3 public and 4 private) met the inclusion criteria while 1 was excluded due to suspension of AST in the laboratory during the study period.

\section{Evaluation of critical control points in the AST process}

\section{Risks related to materials (samples and reagents)}

The criticality index (CI) was $\geq 6$ for the inoculum suspension at 6 of the 7 laboratories (LBM1, LBM2, LBM3, LBM4, LBM5 and LBM7). The CI was also $\geq 6$ for the antibiotic disks at all the laboratories (LBM1 to LBM7). The CI score was 6 for the culture media at 6 of the 7 laboratories (LBM1, LBM2, LBM3, LB M5, LBM6 and LBM 7). However, the CI was $<6$ for bacterial identification in all 7 laboratories (Fig 1)

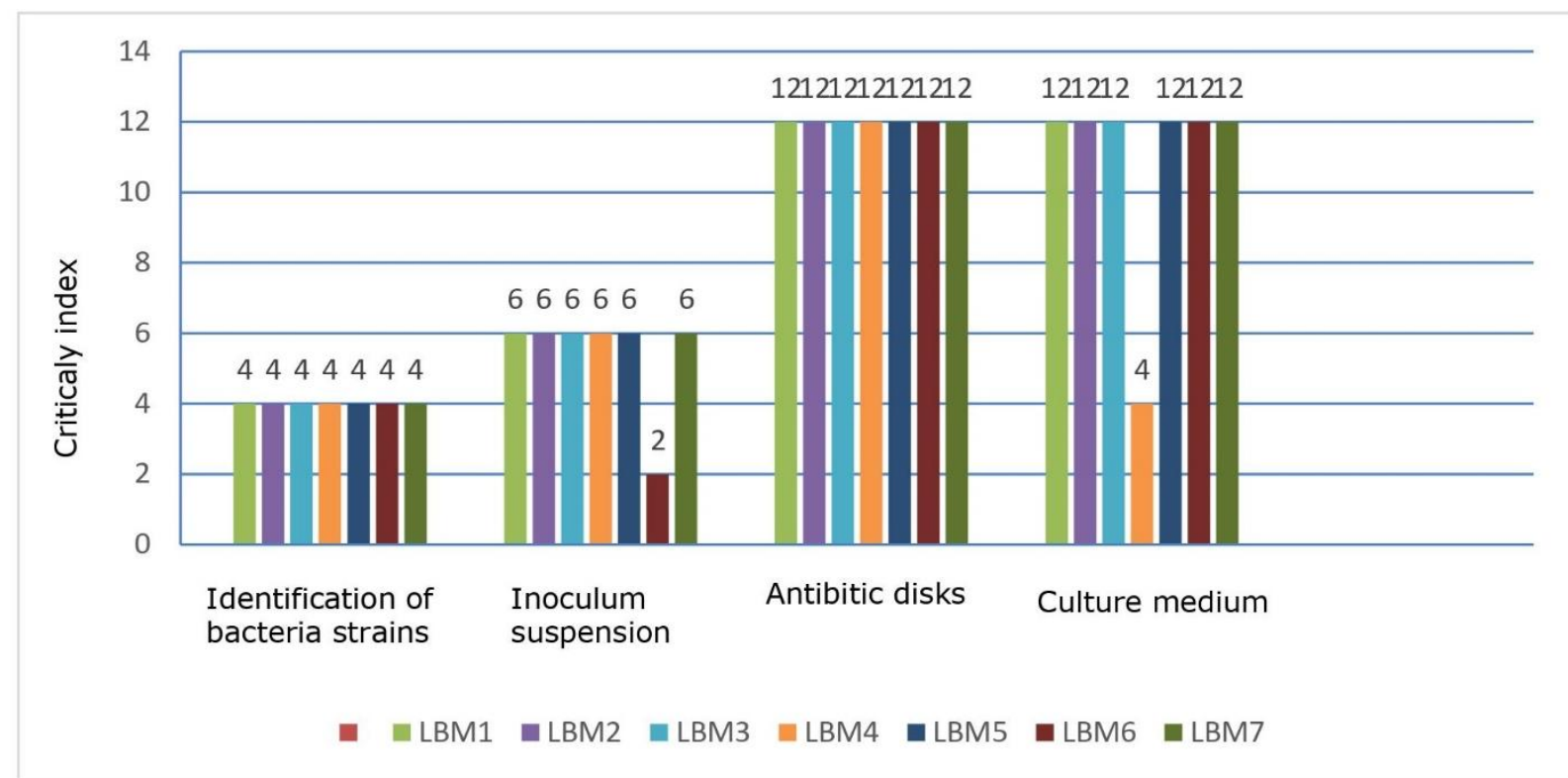

Fig 1: Criticality index of the material (sample and antibiotic discs) used by the different laboratories in the antibiotic susceptibility testing process 


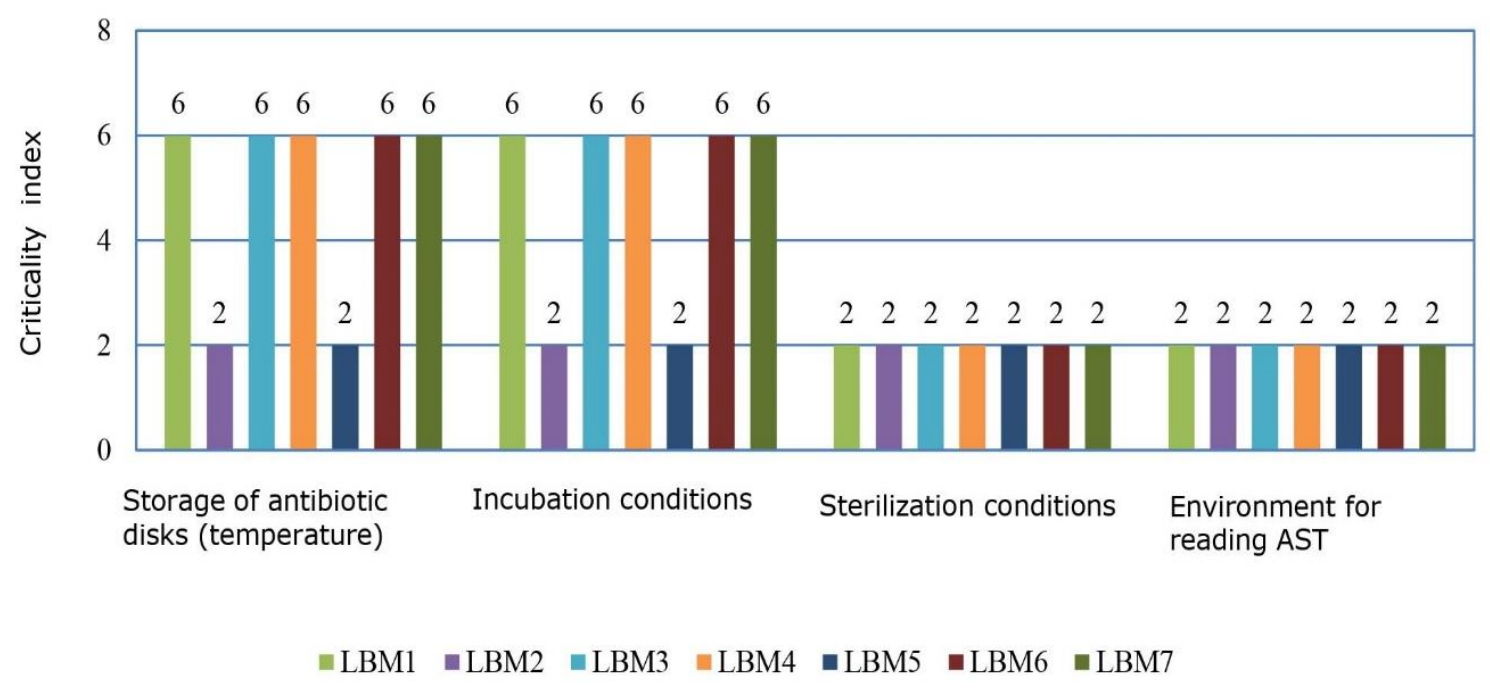

Fig 2: Criticality index of the environment (storage and room temperatures) used by the different laboratories in the process of performing the antibiogram

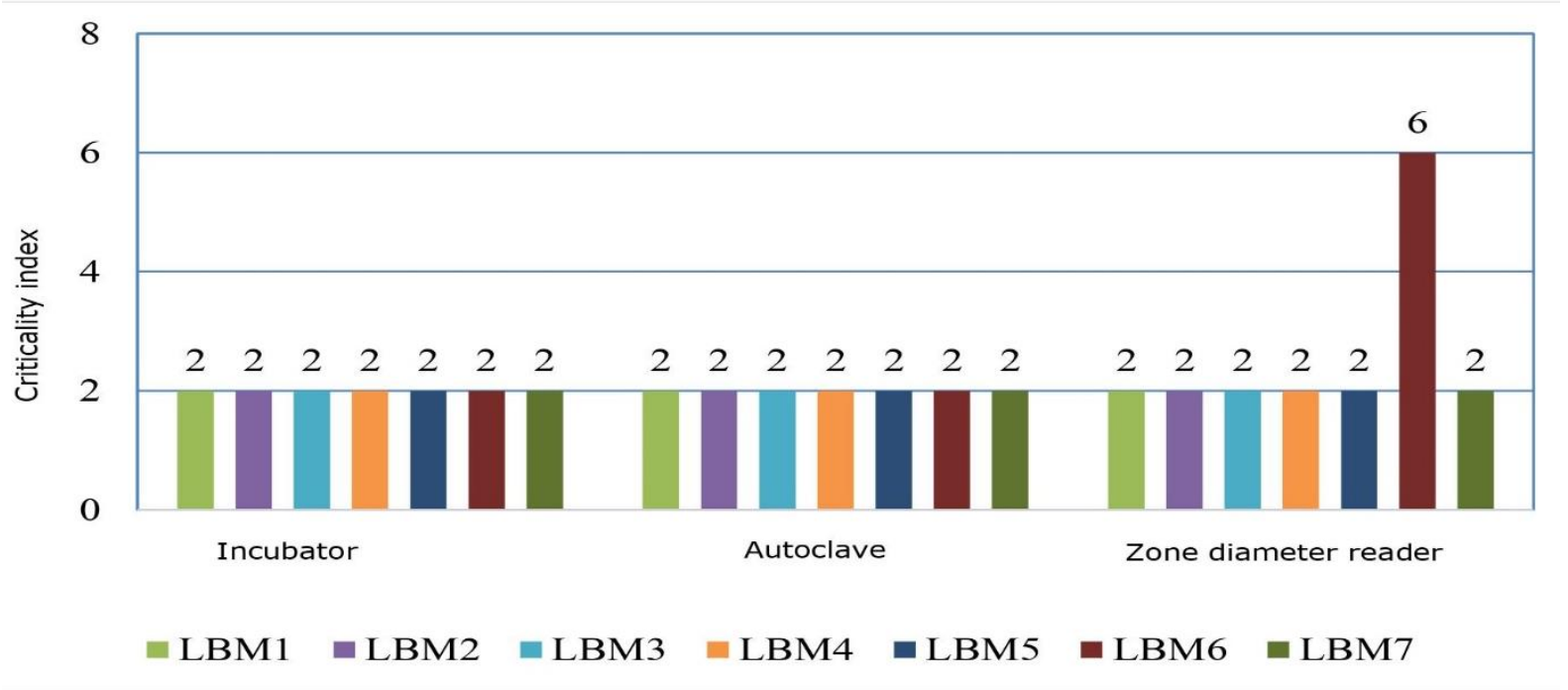

Fig 3: Criticality index of the equipment used by the different laboratories in the AST process

\section{Risks related to media}

The CI score was 6 for storage and incubation conditions at 5 of the 7 laboratories (LBM1, LBM3, LBM4, LBM6 and LBM7), while the CI score for sterilization and environmental condition for reading of AST was $<6$ at all the 7 laboratories (Fig 2).

\section{Risks related to AST materials}

The CI score of 6 for AST reader was reported for only one laboratory (LBM6) while the CI score for incubator and autoclave was $<6$ for all the laboratories (Fig 3)

\section{Risks related to AST method and procedure} The CI score for AST procedure, qua- lity control and reading of the inhibition diameters was 6 at all the laboratories (LBM1 to LBM7) while the CI score of AST result reporting was 6 for 6 of the 7 laboratories (LBM1, LBM3, LBM4, LBM5, LBM6 and LBM7) (Fig 4).

\section{Risks related to the workforce}

The CI score for the choice of antibiotic disks used by the laboratory staff was 9 $(\geq 6)$ for 4 of the 7 laboratories (LBM1, LB M3, LBM5 and LBM6) while the CI score for staff qualification was $<6$ for all the laboratories (Fig 5). 


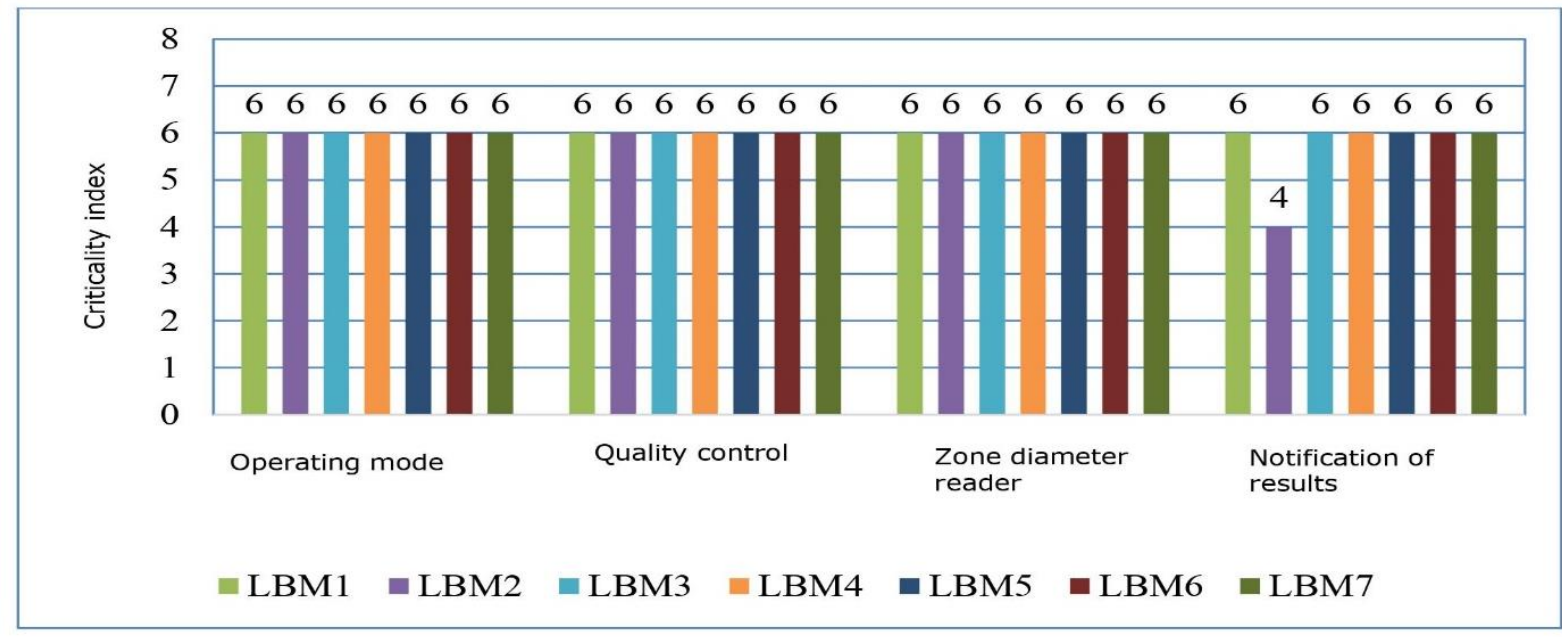

Fig 4: Criticality index of the method used by the different laboratories in the AST process

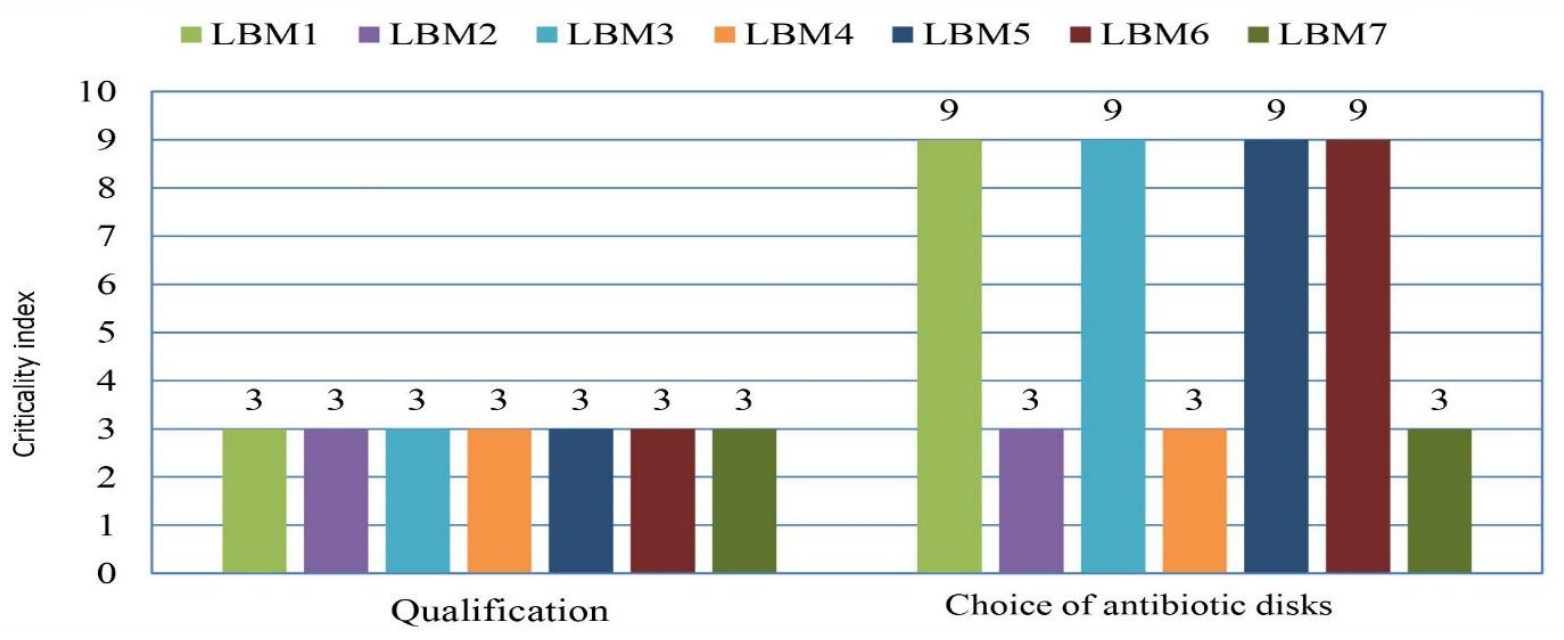

Fig 5: Criticality index of the manpower used by the different laboratories in the antibiogram process.

\section{Evaluation of AST performance}

Essential matches (EC) and measurement errors of diameters of inhibition by the laboratories for reference strains $\mathrm{S1}$ and $\mathrm{S2}$

The essential measurement agreement of the diameters of the inhibition zones for E. coli (S1) and S. aureus (S2) strains was $100 \%$ for LBM3. The frequencies of inhibition zone diameter measurement errors in excess or by default on the reference strain of E. coli (S1) were $52 \%, 50 \%, 42 \%$, $40 \%, 32 \%$ and $28 \%$ for LBM2, LBM4, LBM5, LBM6, LBM4, LBM7, and LBM1, respectively. For the reference $S$. aureus (S2) strain, the frequencies of over- or under-inhibition zone diameter measurement errors were $100 \%$,
$40 \%, 32 \%, 29 \%, 29 \%$, and $20 \%$ for LBM4, LBM6, LBM1, LBM2, LBM5, and LBM7, respectively (Fig 6).

Types of errors in inhibition zone diameters reported by laboratories for reference strains S1 and S2

On strain S1, all (100\%) of the inhibition zone diameter errors encountered at LBM1, LBM4, LBM5 and LBM7 were default errors. Similarly, errors made by the laboratories LBM2 and LBM7 on the diameters of the inhibition zones of the S2 strain were errors by excess. On strain S2, all (100\%) of the inhibition zone diameter errors encountered at LBM2 and LBM7 were excess errors (Fig 7). 
Errors Essentials discrepanies

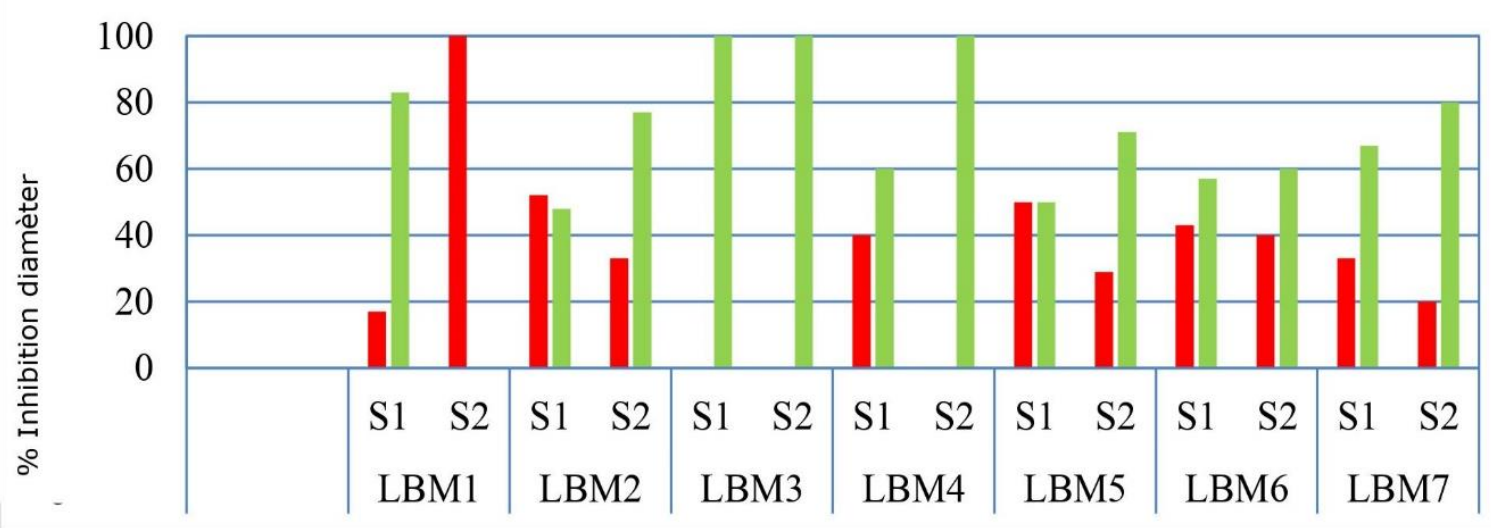

Fig 6: Distribution of essential concordance $(E C)$ and inhibition zone diameter errors by laboratory and reference strains

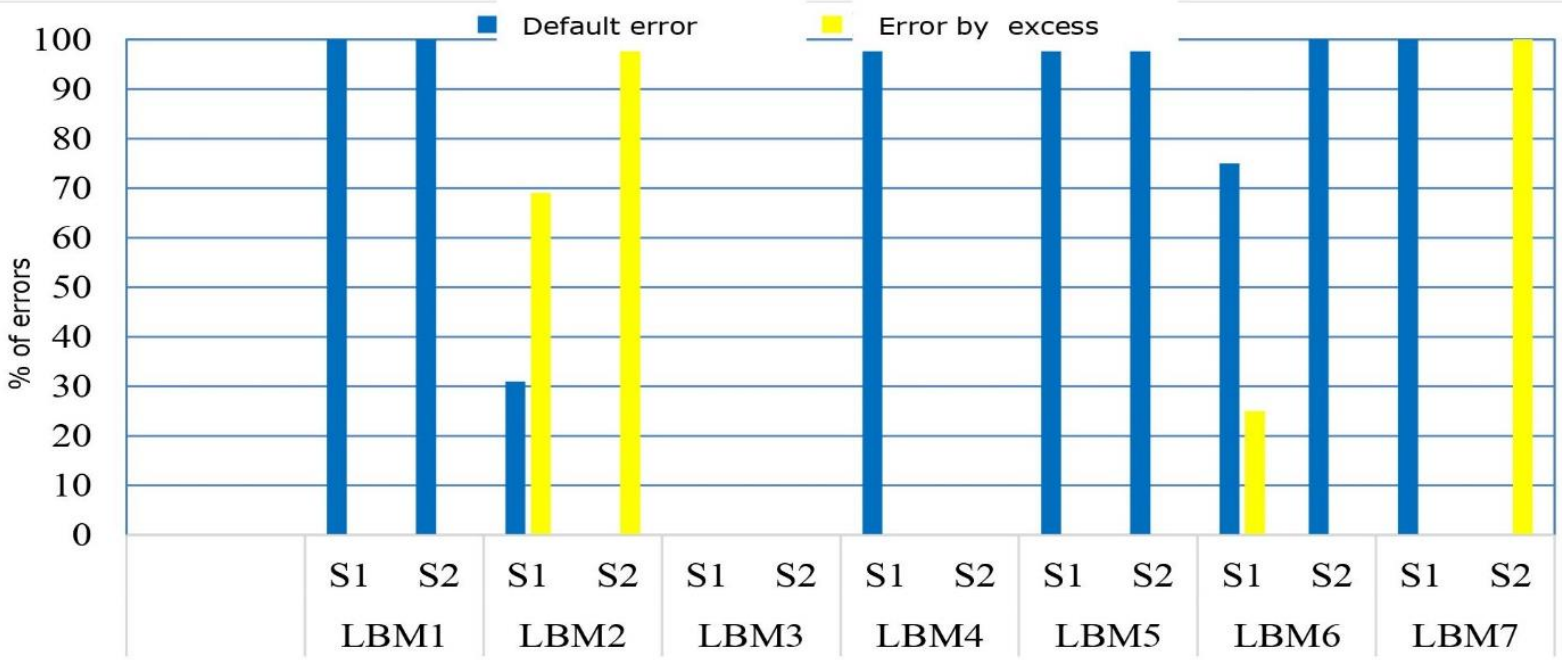

Fig 7: Distribution of the types of errors made on the diameters of the inhibition zones according to the laboratories and the reference strains

S/I/R discrepancies and categorical $S / I / R$ matches reported by the laboratories

The S/I/R categorical discrepancies observed for the reference strain of $E$. coli (S1), were less than $20 \%$ except for LBM5, LBM6, and LBM7 where they were 50\%, $29 \%$, and $33 \%$, respectively. On the reference $S$. aureus strain (S2), the highest S/I/R category discrepancies were found at LBM1 (37\%) and LBM6 (50\%) (Fig 8).

S/I/R categorization discrepancies reported by the laboratories

All $(100 \%)$ the categorization discre- pancies reported by LBM2, LBM3 and LBM7 were major discrepancies while those reported by LBM1 and LBM4 were minor discrepancies (Fig 9).

Antibiotic discs with $S / I / R$ categorization discrepancy reported by laboratories for reference strains S1 and S2

The antibiotics most affected by the $\mathrm{S} / \mathrm{I} / \mathrm{R}$ misclassification were amoxicillin (80 $\%$ ), amoxicillin + clavulanic acid (70\%), trimethoprim (100\%) and vancomycin (100\%) (Fig 10). 
Discordance Categorical concordance (CC)

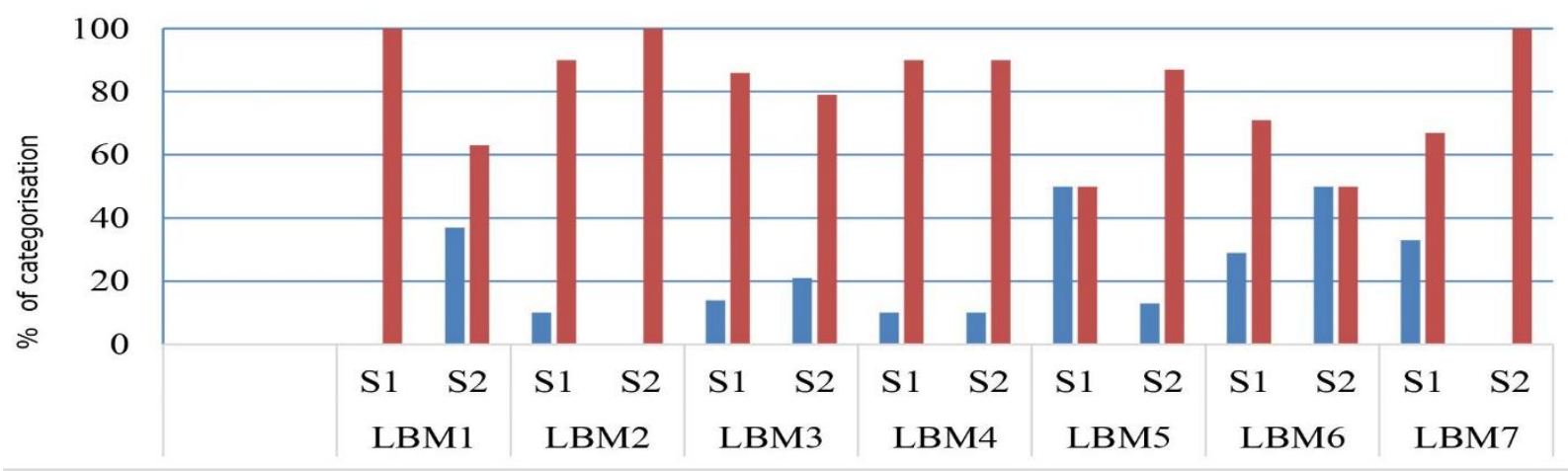

Fig 8: Distribution of S/I/R categorical mismatches and matches by laboratory and reference strain
- Very major dicrepany
- Major discrepany
- Minor discrepany

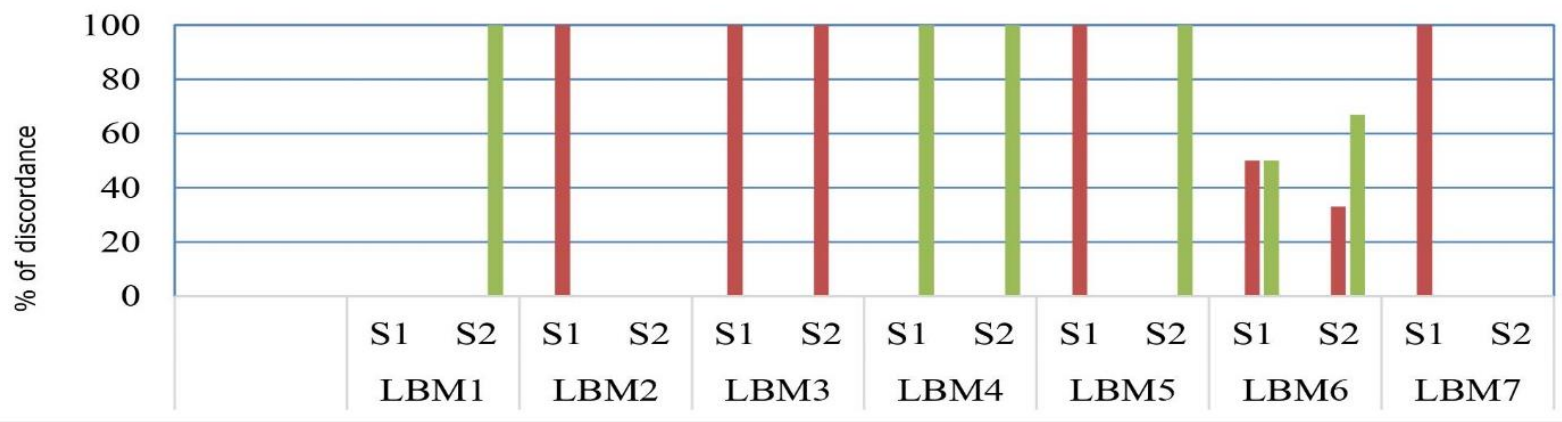

Fig 9: Distribution of S/I/R categorization mismatches by laboratory and reference strains

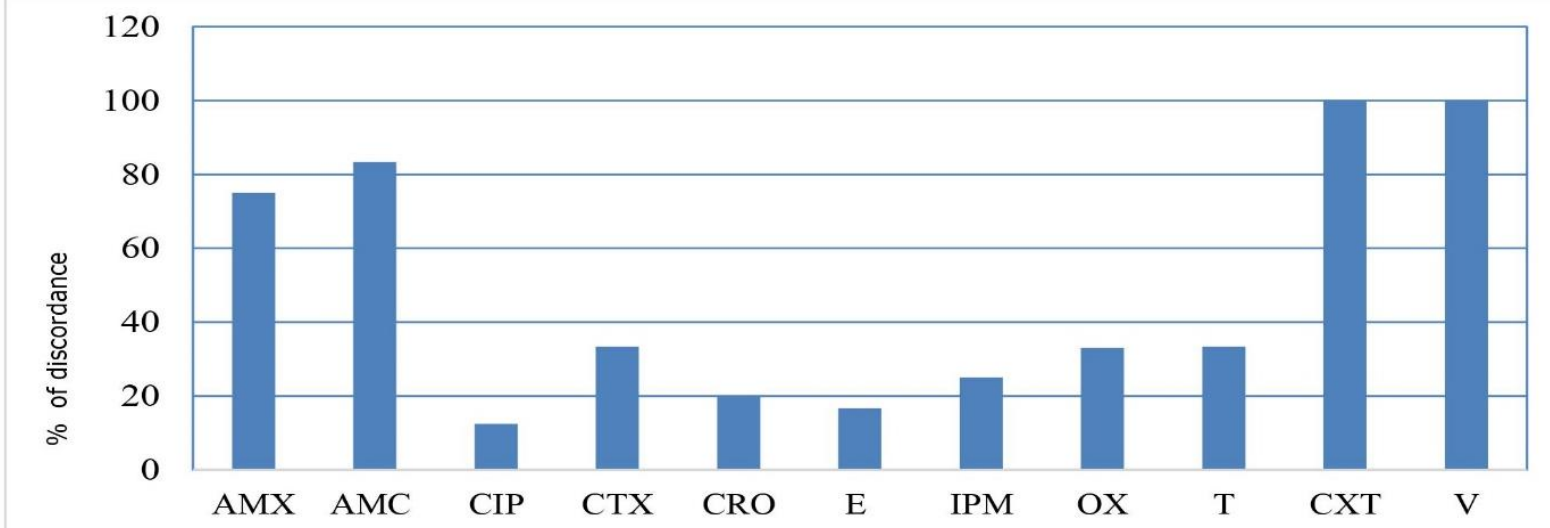

$\mathrm{AMX}=$ Amoxicillin $; \mathrm{CIP}=$ Ciprofloxacin $; \mathrm{CRO}=$ Ceftriaxone $; \mathrm{IPM}=$ Imipenem; $\mathrm{AMC}=$ Amoxicillin + clavulanic acid $; \mathrm{CTX}=\mathrm{Cefotaxime} ; \mathrm{E}=$ Erythromycin; $\mathrm{OX}=$ Oxacillin; $\mathrm{T}=$ Tobramycin; $\mathrm{CXT}=$ Trimethoprim + sulfamethoxazole; $\mathrm{V}=$ Vancomycin

Fig 10: Distribution of S/I/R categorization mismatches by antibiotic disk

\section{Discussion:}

At the material level (sample and reagents) evaluation, inoculum was not standardized in most laboratories because they did not have a standardized method of inoculum preparation. This was similarly reported by Salou et al., (6) in 2016 in Lomé laboratories in Togo. According to Weber (7), "poorly controlled inoculum is a common error especially if a densitometer is not used for calibration". Non-standardization of the inoculum could have a very important impact on the final result of the antibiotic susceptibility test, as heavy inoculum leads to smaller zone diameters and vice versa (8). Thabaut et al., (9) demonstrated that poor standardization of the inoculum resulted in a large variability in the diameters of the inhibition zones for the same bacterial strain. 
Moreover, according to the work of Weber et al., (7) in France excessively heavy inoculum gave results that affected all antibiotics, particularly beta-lactams and glycopeptides, giving falsely intermediate or resistant results. In this study, only one of the seven laboratories controlled the agar thickness according to the EUCAST recommendations ( $4 \mathrm{~mm}$ $\pm 0.5 \mathrm{~mm}$ ) (8). The lack of agar thickness control could lead to inhibition zone diameters outside the performance limits (10). At the environmental level (storage and room temperatures) in this study, 5 of 7 laboratories did not comply with the conditions for storage of disks and culture media and incubation of culture media for AST. These risks were mainly due to the fact that these laboratories did not have mapping or metrological records of the cold chambers or oven. Discharge of the disks leads to reduced inhibition zones and is a common source of errors (8). On hardware evaluation, the risk of error factors related to the materials used in the process of performing the AST i. e. procedures for using ovens, autoclaves and inhibition diameter readers, were relatively well controlled by the laboratories. Nevertheless, we found that one of the laboratories (LBM6) had a very high CI compared to its inhibition zone diameter reader. A survey carried out in this laboratory showed that it did not use any instrument to read the inhibition zone diameters, but only read this with the naked eye. This result confirmed the one reported by Védy et al., (11) in France, which showed that visual reading of AST was subject to variation depending on the eye of the operator.

With regard to evaluation of the performance of AST, all the laboratories had high criticality indexes. This could be due to the lack of standard operating procedures (SOPs) for performing AST, lack of quality control, and absence of double reading by technicians/biologists. In this respect, our results corroborated those of Védy et al., (11) in France, which reported that AST procedure was a risk factor for error and AST results were likely to fluctuate between different technicians on workstation if uniform AST procedures were not applied by all staff.

At the level of the workforce, we discovered that all the laboratories used skilled workforce, but the risk of error was mainly due to the choice of antibiotic disks used by the technicians, which did not correspond to the disks recommended specifically for the performance of AST. Our results justify the use of a single reference system across the country in order to harmonize practices.

Considering accuracy of the results of AST on the reference strains, most of the laboratories reported inhibition zone dia- meters beyond acceptable reference limits for one or both strains. This could be attributed to the fact that no risk factor for error was fully controlled by the laboratories. The LBM3 laboratory that did not report zone diameters out of bounds for either of the two strains also did not have full control of the risk factors. The results from this laboratory were due to the wrong choice of antibiotics tested on the different strains. The errors made by LBM1, LBM4, LBM5 and LBM7 on the diameters of the inhibition zones for the reference (wild) E. coli ATCC 25922, which is a susceptible strain, were all default errors. This type of error could be due to too thick agar, too heavy inoculum, or insufficient loading of the antibiotic disks. Insufficient disk loading could be due to manufacturing error and storage conditions such as temperature or exposure to light or humidity (11). Furthermore, when the inoculated culture boxes are left at laboratory temperature too long before the antibiotic disks are deposited, the bacteria could start to grow, leading to a false decrease in the size of the inhibition zones (7)

The errors made by LBM2 and LBM7 on the inhibition zone diameters of the low beta-lactamase producing $S$. aureus reference strain ATCC 29213 were all oversized errors. An excess error would be much more problematic than a default error, as it predisposes to a higher risk of treatment failure (11). According to the 2018 EUCAST guideline/standard (8), culture boxes should ideally be incubated within 15 minutes after disk deposition, but no longer than $\mathbf{3 0}$ minutes. If they are left at room temperature after disk deposition, the pre-diffusion of antibiotics would result in falsely enlarged inhibition zones. The errors on the inhibition zone diameters of the reference strains made evident the misclassification inconsistencies that we found in the results from all the laboratories. All categorization discrepancies reported by LBM2, LBM3 and LBM7 were major discrepancies, i. e. reported as resistant $(R)$ when the strain was susceptible (S). This type of discrepancy in result can lead to selection of inappropriate antimicrobials for effective treat ment from which the patient could have benefited, but this will not expose the patient to the risk of therapeutic failure. These discrepancies were due to default errors, the sources of which could be too thick agar, excess inoculum, or insufficient loading of the antibiotic disks.

The antibiotic disks most affected by the discordances were; amoxicillin, amoxicillin + clavulanic acid, trimethoprim, and vancomycin, as well as cefotaxime, ceftriaxone, imipenem and oxacillin. This result showed that the affected antibiotic disks were predominantly beta-lactam antibiotics. Accor- 
ding to Adam et al., (12), amoxicillin, cefalotin, amoxicillin + clavulanic acid, and ticarcillin + clavulanic acid were "fragile" antibiotic molecules and had to be stored permanently at $4^{\circ} \mathrm{C}$ in a desiccant container. Similarly, Weber (7) showed that some disks impregnated for AST were particularly sensitive to humidity. In an open cartridge stored at $+4^{\circ} \mathrm{C}$, even in a dispenser containing a desiccant, the antibiotic load loss of a disk was very rapid. For this reason, they recommended that an open cartridge should not be stored for more than 5 days in the refrigerator and that cartridges of particularly sensitive antibiotics, such as beta-lactams should be stored at $-20^{\circ} \mathrm{C}$.

In this study, we noted default errors in ciprofloxacin, which were similar to those reported by King and Brown (10) in their study on quality assurance of disk diffusion sensitivity tests. Fluoroquinolones are known to be inactivated by prolonged exposure to light. From our study, we showed that the discrepancies presented by antibiotic disks were mostly major discrepancies and ranged from 75 to $100 \%$ for amoxicillin, amoxicillin + clavulanic acid, trimethoprim, vancomycin, cefotaxime, imipenem and oxacillin. These findings are similar to those reported by Dougnon et al., (3) in Benin in 2016.

This study has some limitations. The reference strains used by the laboratories in our study received special treatments with respect to AST performance, different from those routinely performed in the standard practice. There was also the possibility of bias in the comparison of results from different laboratories, because the nature and number of antibiotic disks tested per strain differed between the laboratories. However, the findings met the set objectives of our study.

\section{Conclusion:}

The findings of our study showed that the criticality index was high $(\geq 6)$ for most of the critical points related to material, medium, equipment, method, and workmanship at the level of the laboratories assessed. Major and/or minor categorization discrepancies occurred at the level of all laboratories for both reference strains used. Corrective actions are required by these laboratories to improve their performance.

Evaluation of the quality of AST practices should be formalized, carried out periodically and extend to all laboratories in Niger. This could be done within the framework of surveillance of antibacterial resistance, with standardization of the performance of AST at the national level.

\section{References:}

1. Bhat, V., and Vira, H. Quality Control Issues in Antibiotic Susceptibility Testing by Disc Diffusion Technique. Clin Infect Dis. 2018; 2 (1): 104.

2. Société Française de Microbiologie. Recommandations du Comité Qualité (QUAMIC) de la Société Française de Microbiologie. Paris. 2016: 327.

3. Dougnon, T. V., Johnson, R. C., Bankolé, H. S., et al. Évaluation de la Performance de Trois Marques de Disques d'Antibiotiques Vendues au Benin. Health Sciences and Diseases. $2016 ; 17(4)$ : 71-78.

4. Annaix, V., Rogowski, J., Joyau, M., Jaouën, $E$. Gestion des non-conformités au laboratoire de biologie médicale : application aux non-conformités des prélèvements pour l'année 2009. Ann Biol Clin. 2011; 69: 363-372.

5. Naoui, H., Boumhil, L., Bouchrik, M., et al. Mise en place des recommandations du GBEA dans la phase pré-analytique du Laboratoire de Parasitologie à I'Hôpital Militaire d'Instruction Mohamed V de Rabat. Journal de Biologie Médicale. 2018; 27 (7): 200-203

6. Salou, M., Kantchire, Y., Dossim, S., et al. Audit diagnostic de la pratique de l'antibiogramme dans quatre laboratoires de biologie médicale à Lomé, Togo. In: Livre des résumés 048/CO-2/FIBAfric 2018.

7. Weber, M. Les pièges de I'antibiogramme. Revue Française de Laboratoires. 2003; 352: 21-26.

8. Société Française de Microbiologie. Détermination de la sensibilité aux antibiotiques. In : CASFM / EUCAST : Société Française de Microbiologie. 2018; 2: 7-23.

9. Thabaut, A., and Durosoir, J. L. L'antibiogramme: Méthodes classiques et Méthodes automatisées. Med Mal Infect. 1979; 9: 490-495.

10. King, A., and Brown, D. F. Quality assurance of antimicrobial susceptibility testing by disc diffusion. ] Antimicrob Chemother. 2001; 48: 71-6.

11. Védy, S. Contrôle de qualité interne en antibiologie: retour d'expérience. Ann Biol Clin. 2012; 70 (3): 341352.

12. Adam, J. M. Le point sur I'antibiogramme au laboratoire de bactériologie médicale. Rev Francoph Lab. 2005; 375: 50-54. 